\title{
Factors Associated with Echinococcosis-Induced Perioperative Anaphylactic Shock
}

\author{
Jianrong Ye, Qin Zhang, Yan Xuan, Siyu Chen, Long Ma, Yongqiang Zhang, Hong Zheng* \\ Department of Anesthesiology, First Affiliated Hospital of Xinjiang Medical University, Urumqi 830054, P. R. China
}

\begin{abstract}
This retrospective case-control study explored the factors associated with anaphylactic shock during surgery for cystic echinococcosis (CE) at the First Affiliated Hospital of Xinjiang Medical University between October 2008 and September 2013. Patients who suffered from anaphylactic shock $(n=16)$ were age-matched $3: 1$ to patients who did not $(n=43)$. Multivariate analysis suggested that IL-4 levels (odds ratio $=1.096 ; 95 \%$ confidence interval $=1.015-1.185$; $P=0.02$ ) and cyst size (odds ratio $=3.028,95 \%$ confidence interval $=1.259-7.283, P=0.013$ ) were independently associated with $\mathrm{CE}$-induced perioperative anaphylactic shock. Using the receiver operating characteristic (ROC) curves and a cut-off value of $415.7 \mathrm{ng} / \mathrm{ml}$, IL-4 showed an area under the ROC (AUC) of 0.926 , sensitivity of $75.0 \%$, and specificity of $97.7 \%$. Using a cut-off value of $7.8 \mathrm{~cm}$, cyst size showed an AUC of 0.828 , sensitivity of $81.3 \%$, and specificity of $76.7 \%$. In conclusion, results suggest that levels of IL-4 and cyst size were independently associated with echinococcosis-induced perioperative anaphylactic shock. These results could help identifying patients with echinococcosis at risk of anaphylactic shock in whom appropriate prophylaxis could be undertaken.
\end{abstract}

Key words: Echinococcus granulosus, cystic echinococcosis, hydatid cyst, anaphylaxis, surgery, zoonotic infectious disease

\section{INTRODUCTION}

Patients with cystic echinococcosis (CE) are often asymptomatic, and CE is frequently an incidental finding [1]. Complications can be serious in cases of cyst rupture, which may cause secondary infection and anaphylactic shock. Anaphylactic shock after cyst rupture is induced by cystic fluid overflow due to surgery, trauma, or spontaneous cyst rupture, and the incidence is as high as $2.0 \%$ in clinical practices [2,3]. Inappropriate treatment could result in serious outcomes and even death [4]. Anaphylactic shock is associated with inter-individual differences and hypersensitivity, while the severity and speed of anaphylactic shock is associated with the amount of specific antibodies. The levels of antibodies vary with the size, number, and site of Echinococcus infection [5]. The anaphylactic shock induced by echinococcosis can be cytotoxic and is mainly the result of synergistic effects of type 1 immediate hypersensitivity and endotoxic shock [4,6-9]. Large amounts of

- Received 10 May 2016, revised 27 July 2016, accepted 11 September 2016.

*Corresponding author (hzhengxj@sina.com)

(c) 2016, Korean Society for Parasitology and Tropical Medicine

This is an Open Access article distributed under the terms of the Creative Commons Attribution Non-Commercial License (http://creativecommons.org/licenses/by-nc/4.0) which permits unrestricted non-commercial use, distribution, and reproduction in any medium, provided the original work is properly cited. vasoactive substances are found in $\mathrm{CE}$ and could induce vasomotor dysfunction and result in peripheral circulatory failure.

However, the clinical presentations and immunological characteristics of anaphylactic shock that is experienced by some patients are substantially different from type 1 immediate hypersensitivity [9]. Therefore, the usual methods for type 1 immediate hypersensitivity in the treatment of anaphylactic shock induced by echinococcosis have limited success, and may even affect the prognosis of the patients, but available data are limited $[4,10]$. Anaphylactic shock induced by echinococcosis during operation is generally unanticipated $[10,11]$. In addition, the types and levels of specific antibodies released from the cysts are changing with disease course; these specific antibodies play critical roles in echinococcosis-induced anaphylactic shock [12].

The diagnosis of echinococcosis is relatively straightforward using various imaging methods [13], but it may be difficult to predict which patients will develop anaphylaxis $[4,13,14]$. In the present study, we retrospectively analyzed the clinical and immunological data of patients with echinococcosis who developed anaphylactic shock in the perioperative period to identify the associated factors and to provide some evidence for prevention and treatment of this complication. 


\section{MATERIALS AND METHODS}

\section{Patients}

This was a retrospective case-control study performed using a subgroup from a total of 1,426 surgically-treated patients with echinococcosis who were hospitalized at the First Affiliated Hospital of Xinjiang Medical University between October 2008 and September 2013. These patients were identified from the hospital's medical database. All patients presenting with a parasitic infection were prospectively entered in this database. Among these 1,426 patients, 18 suffered from anaphylactic shock due to cystic fluid outflow during surgery, 2 cases of which missing data for the cyst size were excluded. For each of these patients, 3 age-matched controls $(n=43)$ with pulmonary or hepatic echinococcosis and who were found with cystic fluid outflow during the operation but did not develop anaphylactic shock were selected from the patients with echinococcosis.

In this study, all patients were with Echinococcus granulosus. Patients who met 1 or more of the following criteria were excluded: 1) with other co-infections, including viral hepatitis, AIDS, or syphilis; 2) a history of anaphylactic shock from any other cause; or 3) any chronic or acute organ diseases.

Anaphylactic shock was diagnosed according to the criteria by Sampson et al. [15] from the National Institute of Allergy and Infectious Diseases, USA. In brief, patients with the following symptoms within several minutes to several hours were diagnosed with anaphylactic shock: 1) blood pressure sharply decreased to $<80 / 50 \mathrm{mmHg}$ after cystic fluid outflow or systolic pressure decreased by more than $30 \%$ of the basal blood pressure; and 2) allergy-related symptoms, including lesions of the skin and mucosa (wheal, pruritus all over the body, and oral edema) and airway obstruction (such as asphyxia, asthma, tracheospasm, stridor, and reduced peak expiratory flow before or at the same time as the blood pressure decrease.

This study was approved by the ethics committee of the First Affiliated Hospital of Xinjiang Medical University, China.

\section{Surgery}

The surgical methods included endo-cystectomy, extra-cystectomy, and endo- plus extra-cystectomy [15], which was selected by the surgeon according to each patient's conditions. The surgical principle was to remove the endocyst, to avoid spillage of cyst contents, to resect the residual extra-cyst lumens, and to prevent infection [2]. The specific surgical approach was determined by the size of the cyst, and the presence or absence of biliary fistula, inflammation, or calcification.

For example, endocystectomy is the most commonly used approach for hepatic CE. After surgical exposure of the hepatic $\mathrm{CE}$, the incision and surrounding organs are protected using a moistened gauze pad overlapped by a layer of gauze containing 10\% hypertonic saline solution (hypertonic saline solution can kill the larva in the cyst [15]). When the pericyst, in particular those with a high tension, is opened, varying degrees of cyst content spillage will occur. In the absence of biliary fistula, $10 \%$ hypertonic saline solution is re-infused to kill the larva and is aspirated 15 min later. After such infusion and aspiration is repeated for 2-3 times, fluids inside the cyst should be maximally drained. Patients who developed perioperative anaphylactic shock were under continuous monitoring by the anesthesiologists.

\section{Data collection}

Demographic and immunological data of 59 patients (16 with anaphylactic shock and 43 without anaphylactic shock) were collected from the standard questionnaires that are filled for all patients with suspected CE at hospital admission, and analyzed. Demographic data (age, sex, height, weight, ethnicity, blood type, history of allergy, place of residence, time of contact with dogs, cows or sheep, and family history of allergy), clinical symptoms (rash, stridor, the lowest blood pressure at shock, the highest airway pressure, hypoxemia, oxygen saturation at the lowest pulse, and arrhythmia), laboratory examinations (anti-EgCF, anti-EgP, anti-EgB, and anti-Em2), blood routine examination (blood cell counts, hemoglobin, and hematocrit), biochemical examinations (total protein, albumin, globulin, A-albumin, a1-globulin, a2-globulin, $\beta 1$-globulin, $\beta 2$-globulin, and $\gamma$-globulin), imaging data (type of imaging examination, site, number, and size of cysts, number of daughter cysts, rupture of cyst, infection, and imaging diagnosis), and immunological examinations (IgE, IgG, IgG1, IL-4, IL-10, IFN- $\gamma$, and TNF- $\alpha$ ) were retrieved from the medical charts. All laboratory tests were performed the day before surgery. These data/tests were all standard data that were documented when patients with suspected CE visited our hospital.

IL-4 (sensitivity: $2 \mathrm{ng} / \mathrm{L}$; detection range: 16.6-1,000 ng/L), IL-10 (sensitivity: 7 pg/ml; detection range: 15.6-1,000 pg/ml), INF- $\gamma$ (sensitivity: $4 \mathrm{ng} / \mathrm{L}$; detection range: $15.6-1,000 \mathrm{ng} / \mathrm{L}$ ), TNF- $\alpha$ (sensitivity: 4 pg/ml; detection range: 15.6-1,000 $\times 10^{-6}$ $\mu \mathrm{g} / \mathrm{ml}$ ), IgE, IgG, and IgG1 (for all 3 items, sensitivity: $1.0 \mathrm{ng} /$ 
$\mathrm{ml}$; detection range: $0-800 \mathrm{ng} / \mathrm{ml}$ ) were tested using ELISA kits (BD Biosciences, San Jose, California, USA), according to the manufacturer's instructions.

Anti-EgCF, anti-EgP, anti-EgB, and anti-Em2 were tested using the Dot Immunogold Filtration Assay (DIGFA) kit for human echinococcosis (Xinjiang Beisiming Biotechnology Development Co., Urumqi, Xinjiang) (catalog \# Q/XZF0022015). This kit has been described by Feng et al. [16]. The membrane has 4 dots; A, B, C, and D (Supplementary Fig. 1) that are coated with hydatid cystic fluid antigen, scolex antigen, semi-purified cystic fluid antigen, and alveolar hydatid specific antigen Em2, respectively. In the center of the nitrocellulose membrane, there is a dot coated with normal serum as quality control (Supplementary Fig. 1). Briefly, 2 drops of whole blood were taken from the earlobe and mixed with 2 drops of potato agglutinin, diluted with 3 drops of solution A, and transferred to the wells of the reaction plate. The plate was washed with 3 drops of solution B. The background signal was washed away with 3 drops of solution B. The plate was then read. Red dots in the corners of the blot were considered positive; otherwise, it was negative (Supplementary Fig. 2). Based on the intensity of the color, positive results could be classified into + (if the color is close to that of the quality control spot in the center of the blot), ++ (if the color is the same as the quality control spot), +++ (if the color is more intense than the quality control spot). The positive initial screening was determined by positive signals in wells A and B. E. granulosus was determined by positive signals in wells A, B, and C. Alveolar echinococcosis was determined by positive signals in wells $\mathrm{A}$, $\mathrm{B}$, and $\mathrm{D}$.

The cyst size was recorded, and the cysts were classified according to the WHO imaging-based classification $[17,18]$. CE1 (single cyst); the hydatid cyst if filled with water-like fluid. CE2 (multiple cysts); multiple small ball-shaped dark images or halo can be seen in the dark area of the mother cyst, which forms the characteristic picture of "cyst in cyst". CE3 (broken inner cyst); when the inner cyst broke, fluid goes to the space in between inner and outer walls and a "lens of cyst" sign is displayed. CE4 (solid cyst); hydatid cyst regresses gradually and shrinks. CE5 (calcification); during the prolonged stage of E. granulosus, the outer wall becomes hypertrophic with calcium deposition or totally calcified.

Treatment data and patient outcomes (including the development, duration, treatments, and outcomes of anaphylactic shock) included: 1) general characteristics (time of echinococ- cosis diagnosis, fever, history of cystectomy, comorbidities, history of anti-echinococcosis medical therapy, operation method, and use of glucocorticoids before cyst isolation); 2) data during the shock (rupture of cysts, outflow of cystic fluid, volume of outflowed cystic fluid, property of the cystic fluid, and the site of outflow), and time between cyst puncture and shock (min); 3) treatments (pure oxygen inhalation, use of epinephrine, use of dopamine, use of adrenocortical hormones, type of fluid therapy, and volume of fluid infusion).

\section{Statistical analysis}

Matching was performed using the database of cases of echinococcosis treated at our hospital. SPSS 19.0 software (IBM, Armonk, New York, USA) was used for statistical analyses. If normally distributed, data are expressed as means $\pm S D$, and were compared using the independent samples $t$-test. If the data were not normally distributed, they were expressed as median (range), and compared using the Mann-Whitney U test. Categorical variables were expressed as proportions, and were compared using the Fisher's exact test or the chi-square test, as appropriate. Multivariate logistical regression analysis was used to identify factors associated with anaphylactic shock.

Selected variables with $P$-values $<0.5$ in the univariate analyses were considered for inclusion in the regression model, but because the sample size was small, categorical variables were not considered for the regression model. Finally, IL-4, IgE, IgG, TNF- $\alpha$, and cyst size were considered for inclusion in the regression model. The discriminative ability to identify anaphylactic shock with risk markers of IL-4 and cyst size was verified using the area under the receiver operating characteristic (ROC) curve (AUC). $P<0.05$ was considered statistically significant.

\section{RESULTS}

\section{Characteristics of the patients}

General characteristics of the patients are presented in Table 1. Eight males and 8 females were selected as the shock cases, while 20 males and 23 females were selected as the control group. The age, gender, height, weight, personal and familial allergic history, living with a dog, WHO imaging classification, and number of cysts were comparable between the 2 groups. The levels of globulin, A-globulin, a1-globulin, a2-globulin, $\beta 1$-globulin, $\beta 2$-globulin, $\gamma$-globulin, IgG1, IL-10, IFN- $\gamma$, anti- 
Table 1. Demographic and clinical characteristics of patients with echinococcosis who were treated surgically with or without anaphylactic shock

\begin{tabular}{|c|c|c|c|}
\hline Parameters & Shock $(n=16)$ & No shock $(n=43)$ & $P$-value \\
\hline Age (year) & $35.5(9-72)$ & $45(8-74)$ & -- \\
\hline $\begin{array}{l}\text { Gender, n (\%) } \\
\text { Male } \\
\text { Female }\end{array}$ & $\begin{array}{l}8(50.0) \\
8(50.0)\end{array}$ & $\begin{array}{l}20(46.5) \\
23(53.5)\end{array}$ & 0.811 \\
\hline Height (cm) & $163(126-178)$ & $165(120-180)$ & 0.804 \\
\hline Weight (kg) & $60(25-107)$ & $67(18-85)$ & 0.447 \\
\hline $\begin{array}{l}\text { Ethnicity, n (\%) } \\
\text { Han } \\
\text { Other }\end{array}$ & $\begin{array}{r}13(81.3) \\
3(18.8)\end{array}$ & $\begin{array}{l}22(51.2) \\
21(48.8)\end{array}$ & $0.036^{*}$ \\
\hline $\begin{array}{l}\text { Allergic history, n (\%) } \\
\text { Yes } \\
\text { No }\end{array}$ & $\begin{array}{r}3(18.8) \\
13(81.3)\end{array}$ & $\begin{array}{c}1(2.3) \\
42(97.7)\end{array}$ & 0.099 \\
\hline $\begin{array}{l}\text { Live with a dog, n (\%) } \\
\text { Yes } \\
\text { No }\end{array}$ & $\begin{array}{r}13(81.3) \\
3(18.8)\end{array}$ & $\begin{array}{r}38(88.4) \\
5(11.6)\end{array}$ & 0.777 \\
\hline $\begin{array}{l}\text { Preoperative diagnosis, n (\%) } \\
\text { Pulmonary echinococcosis } \\
\text { Hepatic echinococcosis }\end{array}$ & $\begin{array}{r}9(56.3) \\
10(62.5)\end{array}$ & $\begin{array}{r}6(14.0) \\
37(86.0)\end{array}$ & $\begin{array}{l}0.003^{\star \star} \\
0.102\end{array}$ \\
\hline $\begin{array}{l}\text { Family history of allergy, n (\%) } \\
\text { Yes } \\
\text { No }\end{array}$ & $\begin{array}{c}1(6.3) \\
15(93.8)\end{array}$ & $\begin{array}{l}0 \\
43(100.0)\end{array}$ & 0.271 \\
\hline $\begin{array}{l}\text { WHO imaging classification } \\
\text { CE1 } \\
\text { CE2 } \\
\text { CE3 }\end{array}$ & $\begin{array}{l}3(18.8) \\
7(43.8) \\
6(37.5)\end{array}$ & $\begin{array}{l}16(37.2) \\
15(34.9) \\
12(27.9)\end{array}$ & 0.377 \\
\hline Cyst size (cm) & $9.36 \pm 2.65$ & $6.50 \pm 1.81$ & $0.001^{* *}$ \\
\hline Number of cysts & $2(1-8)$ & $2(1-8)$ & 0.881 \\
\hline Number of daughter cysts & $7.5(1-16)$ & $5(1-17)$ & $0.010^{*}$ \\
\hline Globulin (g/L) & $34.8(24.8-61.5)$ & $28(19.7-44.6)$ & 0.059 \\
\hline A-globulin (g/L) & 53 (25.9-59.4) & $56(38.6-64.8)$ & 0.147 \\
\hline a1-globulin (g/L) & $3.45(2.4-7.6)$ & $2.4(1.6-7.6)$ & 0.058 \\
\hline a2-globulin (g/L) & $10.5(7.6-15.8)$ & $10.1(6.9-15.8)$ & 0.615 \\
\hline$\beta 1$-globulin (g/L) & $7.6(4.7-11.1)$ & $8.4(4.7-11.2)$ & 0.255 \\
\hline$\beta 2$-globulin (g/L) & $4.2(4.0-6.5)$ & $4.2(3.9,-.5)$ & 0.611 \\
\hline Y-globulin (g/L) & $20.7(18-49)$ & $18.6(12-39)$ & 0.059 \\
\hline $\operatorname{lgE}(\mathrm{ng} / \mathrm{ml})$ & $233.1(112-1,467)$ & $166.3(71-261)$ & $0.010^{*}$ \\
\hline $\lg G(g / L)$ & $377.4 \pm 159.3$ & $247.8 \pm 76.9$ & $<0.001^{\star \star \star}$ \\
\hline $\operatorname{lgG1}(g / L)$ & $102.3(10-327)$ & $96.3(25-148)$ & 0.658 \\
\hline IL-4 (ng/L) & $452.3(341-515)$ & $347.5(140-424)$ & $<0.001^{\star \star \star}$ \\
\hline IL-10 (pg/ml) & $28.8 \pm 11.9$ & $24.0 \pm 7.6$ & 0.069 \\
\hline INF- $\gamma$ (ng/L) & $200.6(99-301)$ & $153.7(83-309)$ & 0.298 \\
\hline TNF-a $(\mu \mathrm{g} / \mathrm{ml})$ & $0.27 \pm 0.20$ & $0.44 \pm 0.22$ & $0.008^{\star *}$ \\
\hline $\begin{array}{l}\text { Anti-EgCF antibody, n (\%) } \\
+ \\
++ \\
+++\end{array}$ & $\begin{array}{r}3(18.8) \\
10(62.5) \\
3(18.8)\end{array}$ & $\begin{array}{r}5(11.6) \\
12(27.9) \\
26(60.5)\end{array}$ & $0.012^{*}$ \\
\hline $\begin{array}{l}\text { Anti-EgP antibody, n (\%) } \\
\quad+ \\
++ \\
+++\end{array}$ & $\begin{array}{r}3(18.8) \\
11(68.8) \\
2(12.5)\end{array}$ & $\begin{aligned} 5 & (11.6) \\
38 & (88.4) \\
0 & (0.0)\end{aligned}$ & 0.054 \\
\hline $\begin{array}{l}\text { Anti-EgB antibody, n (\%) } \\
\quad+ \\
++ \\
+++\end{array}$ & $\begin{array}{r}3(18.8) \\
10(62.5) \\
3(18.8)\end{array}$ & $\begin{array}{r}6(14.0) \\
10(23.3) \\
27(62.8)\end{array}$ & $0.006^{\star \star}$ \\
\hline $\begin{array}{l}\text { Anti-Em2 antibody, n (\%) } \\
- \\
+\end{array}$ & $\begin{array}{r}3(18.8) \\
13(81.3)\end{array}$ & $\begin{array}{c}3(7.0) \\
40(93.0)\end{array}$ & 0.398 \\
\hline Postoperative intubation time (h) & $5(2-8)$ & $0.5(0.15-1.25)$ & $<0.001^{\star \star \star}$ \\
\hline
\end{tabular}

The data are shown as mean $\pm \mathrm{SD}$, median (range), or proportion, as appropriate.

WHO, World Health Organization; anti-EgCF, antibodies against fluid extract of $E$. granulosus; anti-EgP, antibodies against $E$. granulosus protoscolex; anti-EgB, antobodies against $E$. granulosus hydatid cyst fluid lipoprotein antigen; anti-Em2, antibodies against $E$. multilocularis metacestode antigen. ${ }^{\star} P<0.05,{ }^{\star *} P<0.01,{ }^{\star \star \star} P<0.001$. 
EgP antibody, and anti-Em2 antibody were similar between the 2 groups. Of note was that IgG and IL-4 levels were higher in the shock group (both $P<0.001$ ), while TNF- $a$ levels were lower $(P=0.008)$. The frequencies of Han ethnicity $(P=0.04)$ and pulmonary echinococcosis $(P=0.003)$ were higher in the shock group compared with controls; the number of daughter cysts $(P=0.01)$ and postoperative intubation time $(P<0.001)$ were higher in the shock group. Compared with controls, the qualitative evaluation showed lower levels of anti-EgCF $(P=0.01)$ and anti-EgB $(P=0.006)$ antibodies in the shock group. All patients were with E. granulosus. All anaphylactic shocks occurred perioperatively.

\section{Multivariate analysis of the factors associated with perioperative anaphylactic shock}

Because of the small sample size, categorical variables could not be entered into the multivariate model without making it unstable. All continuous variables with $P<0.05$ in univariate analyses were included. Multivariate analysis showed that IL-4 levels, with odds ratio (OR) of 1.096 and 95\% confidence interval (95\% CI) of 1.015-1.185 ( $P=0.02)$ and cyst size, with OR of 3.028 and $95 \% \mathrm{CI}$ of 1.259-7.283 $(P=0.013)$ were independently associated with CE-induced perioperative anaphylactic shock.

\section{$\mathrm{ROC}$ analysis}

The 2 variables identified in the multivariate analysis were tested using the ROC curve method for the prediction of anaphylactic shock during surgery for $\mathrm{CE}$. Using a cut-off value of $415.67 \mathrm{ng} / \mathrm{ml}$, IL-4 showed an AUC of 0.926 , sensitivity of $75.0 \%$, and specificity of $97.7 \%$. Using a cut-off value of 7.8 $\mathrm{cm}$, cyst size showed an AUC of 0.828 , sensitivity of $81.3 \%$, and specificity of $76.7 \%$.

\section{DISCUSSION}

Anaphylactic shock is a serious complication of echinococcosis treatment, but it is difficult to predict whether Echinococcus-infected patients will suffer from this complication during surgical treatment. The aim of this study was to investigate retrospectively the medical records of patients to identify factors associated with anaphylaxis in Echinococcus infection to improve prediction and treatments. This study showed that IgG and IL-4 levels were elevated in the shock group, and that IL-4 levels and cyst size were independently associated with echi- nococcosis-induced perioperative anaphylactic shock.

Alves and Sheikh [19] suggested that the development of anaphylactic shock could be associated with patients' age: children develop food-induced anaphylactic shock more easily, while middle aged and elderly people develop drug-induced anaphylactic shock more easily. Most anaphylactic shock occur in patients over 55 years, and the incidence of anaphylactic shock is significantly lower in patients of ages between 15 and 55 years [20]. Because age is a risk factor for anaphylactic shock to E. granulosus, patients in the present study were matched for age, preventing the observation of the effect of age on toxic anaphylactic shock occurrence.

IgG is involved in anaphylactic shock or even death induced by antigens of parasites alongside IgE [14,21]. IgG accounts for about $75-80 \%$ of all immunoglobulins in the serum, and consists of 4 subtypes, IgG1, IgG2, IgG3, and IgG4 [22]. The concentration of IgG1 is the highest among all IgGs [22]. Allergens are good inducers of IgG1 and IgG4 [22]. The findings of the present study further suggested that pre-surgery IgG levels were higher in the shock group, but there was no association with IgG1 levels. This could be due to the possibility that patients whose immune system is already under a state of activation could be more vulnerable to the detrimental effects of Echinococcus antigens after cystic fluid spilling. However, more studies with larger sample sizes are needed to further investigate whether IgG is involved in the anaphylactic shock induced by echinococcosis. The reactive responses of the body are different against different antigens among different individuals, and the $\mathrm{IgG} / \mathrm{IgE}$ ratio could also be different, which could result in different risks of shock. The univariate analyses showed that the levels of anti-EgCF and anti-EgB antibodies were lower in the shock group compared with controls, but these variables could not be entered in the multivariate model.

Currently, in our center, most surgeries for CE are endo- plus extra-cystectomy, but in order to maximize the protection of the lung tissues, patients with pulmonary hydatid cyst all undergo endocystectomy. The probability of cyst rupture during endocystectomy is high $[15,20,23,24]$. For patients with hepatic $\mathrm{CE}$, endo- plus extra-cystectomy is the commonly used approach. Spillage of cyst contents seldom occur during single pericystectomy, but it can be frequently observed during endocystectomy or endo- plus extra-cystectomy $[15,20,23,24]$. The exact cause of cyst rupture during surgery is generally considered as resulting from the pressure applied to the cyst during surgery, albeit all efforts are made to avoid that. If there are se- 
vere adhesions or if the $\mathrm{CE}$ is superficial, there is a high risk of cyst rupture $[15,20,23,24]$. Another high-risk situation is the decompression of huge and hypertonic CEs [20,24]. In addition, we cannot exclude the possibility that an iatrogenic cyst rupture may have happened, but the exact cause of cyst rupture was not necessarily documented. Nevertheless, the aim of the present study was not to assess the surgical approaches leading to cyst rupture, but to examine the differences in patients who developed an anaphylactic shock after cyst rupture, and those who did not. In the univariate analysis, pulmonary echinococcosis was associated with a higher risk of anaphylactic shock, but the variable could be entered in the multivariate model because of the small sample size. Nevertheless, whether the shock was the result of the cystic fluid spillage due to the surgical technique or the result of specific characteristics of pulmonary echinococcosis remains to be determined.

This study suggests that cyst size was independently associated with echinococcosis-induced perioperative anaphylactic shock. A previous study has shown that large lung hydatid cysts $(>10 \mathrm{~cm}$ ) were more prone to rupture than small cysts, but that the size was not associated with short-term outcomes, but their sample size small and they observed only 3 cases of anaphylactic reaction [25]. In this study, the WHO classification of cysts was not associated with anaphylactic shock after cyst rupture, but all cases were CE1-3, which are all active or transitional diseases that contained active protoscolices. Nevertheless, the CE1-3 classification is based on the morphology of the cysts, which may be of variable sizes $[17,18]$. A possible explanation for the association between cyst size and anaphylactic shock could simply be the larger amount of cystic fluid that could contain a larger amount of antigens, leading to a higher risk of allergic reaction. Additional studies are necessary to address these issues adequately.

A study analyzed the crude fluid content of the cysts in sheep, and has shown that the crude fluid contains an antigenic component that is a specific antigen to IgE [26]. Crude fluid has been shown to modulate the transition of monocytes to dendritic cells in humans, to impair the secretion of IL-12, IL-6, and PGE2 in response to lipopolysaccharides (LPS), and to increase CD14 expression on dendritic cells [6]. The same study also showed that incubation of dendritic cells with GMCSF and IL-4 impaired their ability to secrete IL-12, IL-6, and PGE2 in response to LPS. IL-12 is well known to perpetuate the Th1 response and to inhibit the production of IgE, therefore inhibiting the allergic reaction [27]. PGE2 is a lipid that is produced by the body during immune reactions in an attempt to control the inflammatory process [28]. Therefore, our results suggested that high levels of IL-4 and low levels of TNF- $\alpha$ before surgery, as observed in the shock group, could impair the self-control function of the immune system. However, further studies are necessary to elucidate this point. Particular care should be taken to sample the crude cystic fluid for analysis, which is often difficult when the fluid is spilling, mixing with blood and saline.

This study has some limitations. By nature, a retrospective study may include some bias in respect to the selection of the study subjects, and the controls were selected to be closely matched to the shock cases so this may also have introduced some bias. In addition, because of the retrospective nature of the study, the quality of the data contained in the charts could not be controlled, and the present study was limited to the tests that are routinely performed in patients with CE. Thus, the populations may not be representative. Because anaphylaxis occurs quite rarely, the study population was also small. Because of the small sample size and associated statistical limitations, categorical variables could not be tested in the multivariate model. A previous study by our group reported the characteristics of patients with CE [4], while this current study analyzed biochemical and immunological factors in order to identify risk factors for anaphylactic shock after cyst rupture. Finally, the crude content of the cysts was not analyzed.

In conclusion, IL-4 levels and cyst size were independently associated with echinococcosis-induced perioperative anaphylactic shock. These results could help identifying patients with echinococcosis at risk of anaphylactic shock in whom appropriate prophylaxis could be undertaken.

\section{ACKNOWLEDGMENT}

This study was supported by the National Natural Science Foundation of China (grant no. 81460309).

\section{CONFLICT OF INTEREST}

The authors declare that they have no conflict of interest.

\section{REFERENCES}

1. Wen H, Aji T, Shao YM. Diagnosis and management against the complications of human cystic echinococcosis. Front Med Chi- 
na 2010; 4: 394-398.

2. Yilmaz M, Akbulut S, Kahraman A, Yilmaz S. Liver hydatid cyst rupture into the peritoneal cavity after abdominal trauma: case report and literature review. Int Surg 2012; 97: 239-244.

3. Nunnari G, Pinzone MR, Gruttadauria S, Celesia BM, Madeddu G, Malaguarnera G, Pavone P, Cappellani A, Cacopardo B. Hepatic echinococcosis: clinical and therapeutic aspects. World J Gastroenterol 2012; 18: 1448-1458.

4. Li Y, Zheng H, Cao X, Liu Z, Chen L. Demographic and clinical characteristics of patients with anaphylactic shock after surgery for cystic echinococcosis. Am J Trop Med Hyg 2011; 85: 452-455.

5. Hernández-González A, Muro A, Barrera I, Ramos G, Orduña A, Siles-Lucas M. Usefulness of four different Echinococcus granulosus recombinant antigens for serodiagnosis of unilocular hydatid disease (UHD) and postsurgical follow-up of patients treated for UHD. Clin Vaccine Immunol 2008; 15: 147-153.

6. Kanan JH, Chain BM. Modulation of dendritic cell differentiation and cytokine secretion by the hydatid cyst fluid of Echinococcus granulosus. Immunology 2006; 118: 271-278.

7. Magambo J, Njoroge E, Zeyhle E. Epidemiology and control of echinococcosis in sub-Saharan Africa. Parasitol Int 2006; 55: 193195.

8. Moore KW, de Waal Malefyt R, Coffman RL, O'Garra A. Interleukin-10 and the interleukin-10 receptor. Annu Rev Immunol 2001; 19: 683-765.

9. Vuitton DA. Echinococcosis and allergy. Clin Rev Allergy Immunol 2004; 26: 93-104.

10. Neumayr A, Troia G, de Bernardis C, Tamarozzi F, Goblirsch S, Piccoli L, Hatz C, Filice C, Brunetti E. Justified concern or exaggerated fear: the risk of anaphylaxis in percutaneous treatment of cystic echinococcosis-a systematic literature review. PLoS Negl Trop Dis 2011; 5: e1154.

11. Simpson CR, Newton J, Hippisley-Cox J, Sheikh A. Incidence and prevalence of multiple allergic disorders recorded in a national primary care database. J R Soc Med 2008; 101: 558-563.

12. Zhang W, Ross AG, McManus DP. Mechanisms of immunity in hydatid disease: implications for vaccine development. J Immunol 2008; 181: 6679-6685.

13. Siracusano A, Teggi A, Ortona E. Human cystic echinococcosis: old problems and new perspectives. Interdiscip Perspect Infect Dis 2009; 2009: 474368 .

14. Li Y, Zheng H, Gu M, Cao X, Wen H, Liu Z, Liu T. Comparisons of serum total IgE, IgG, and IgG1 levels in patients with and without echinococcosis-induced anaphylactic shock. Am J Trop Med Hyg 2012; 87: 104-108.

15. Sampson HA, Muñoz-Furlong A, Bock SA, Schmitt C, Bass R, Chowdhury BA, Decker WW, Furlong TJ, Galli SJ, Golden DB, Gruchalla RS, Harlor AD Jr, Hepner DL, Howarth M, Kaplan AP, Levy JH, Lewis LM, Lieberman PL, Metcalfe DD, Murphy R, Pol- lart SM, Pumphrey RS, Rosenwasser LJ, Simons FE, Wood JP, Camargo CA Jr. Symposium on the definition and management of anaphylaxis: summary report. J Allergy Clin Immunol 2005; 115: 584-591.

16. Feng X, Wen H, Zhang Z, Chen X, Ma X, Zhang J, Qi X, Bradshaw $H$, Vuitton D, Craig PS. Dot immunogold filtration assay (DIGFA) with multiple native antigens for rapid serodiagnosis of human cystic and alveolar echinococcosis. Acta Trop 2010; 113: $114-120$.

17. WHO Informal Working Group. International classification of ultrasound images in cystic echinococcosis for application in clinical and field epidemiological settings. Acta Trop 2003; 85: 253-261.

18. Gharbi HA, Hassine W, Brauner MW, Dupuch K. Ultrasound examination of the hydatic liver. Radiology 1981; 139: 459-463.

19. Alves B, Sheikh A. Age specific aetiology of anaphylaxis. Arch Dis Child 2001; 85: 348.

20. Stone SF, Cotterell C, Isbister GK, Holdgate A, Brown SG. Elevated serum cytokines during human anaphylaxis: Identification of potential mediators of acute allergic reactions. J Allergy Clin Immunol 2009; 124: 786-792.

21. Miyajima H, Watanabe N, Ovary Z, Okumura K, Hirano T. Rat monoclonal anti-murine IgE antibody removes IgE molecules already bound to mast cells or basophilic leukemia cells, resulting in the inhibition of systemic anaphylaxis and passive cutaneous anaphylaxis. Int Arch Allergy Immunol 2002; 128: 24-32.

22. Vidarsson G, Dekkers G, Rispens T. IgG subclasses and allotypes: from structure to effector functions. Front Immunol 2014; 5: 520.

23. Kayaalp C, Balkan M, Aydin C, Ozgurtas T, Tanyuksel M, Kirimlioglu V, Akoglu M, Oner K, Pekcan M. Hypertonic saline in hydatid disease. World J Surg 2001; 25: 975-979.

24. Zaharie F, Bartos D, Mocan L, Zaharie R, Iancu C, Tomus C. Open or laparoscopic treatment for hydatid disease of the liver? A 10-year single-institution experience. Surg Endosc 2013; 27: 2110-2116.

25. Usluer O, Ceylan KC, Kaya S, Sevinc S, Gursoy S. Surgical management of pulmonary hydatid cysts: is size an important prognostic indicator? Tex Heart Inst J 2010; 37: 429-434.

26. Zheng H, Xu ZX, Yang GX, Wen H. Recognition of Specific Antigens by Specific IgG and IgE During Anaphylactic Shock Induced by Echinococcus granulosus in Sheep. Int J Automation Comp 2002; 20: 358-360.

27. Kay AB. Allergy and allergic diseases. First of two parts. N Engl J Med 2001; 344: 30-37.

28. Church RJ, Jania LA, Koller BH. Prostaglandin E(2) produced by the lung augments the effector phase of allergic inflammation. J Immunol 2012; 188: 4093-4102. 





Supplementary Fig. 1. Dot Immunogold Filtration Assay (DIGFA) kit for human echinococcosis. The device is made of 3 parts; the plastic capsule, absorbent filler, and nitrocellulose membrane. The kit includes 4 solutions: Solution A for dilution; Solution B for washing; Solution C for colloidal gold-labeled secondary antibody; and STA for potato agglutinin. The membrane has 4 dots (A, B, C, and D) that are coated with hydatid cyst fluid antigen, scolex antigen, semi-purified cyst fluid antigen, and alveolar hydatid specific antigen Em2, respectively. In the center of the NC membrane, there is a dot coated with normal serum for quality control. The membrane is dried at room temperature and then stored at $4^{\circ} \mathrm{C}$ until use. Two drops of whole blood are taken from the earlobe and mixed with 2 drops of potato agglutinin, diluted with 3 drops of solution $\mathrm{A}$, and transferred to the wells of the reaction plate. The plate is washed with 3 drops of solution B, stained with solution $\mathrm{C}$, and washed with 3 drops of solution B again. 

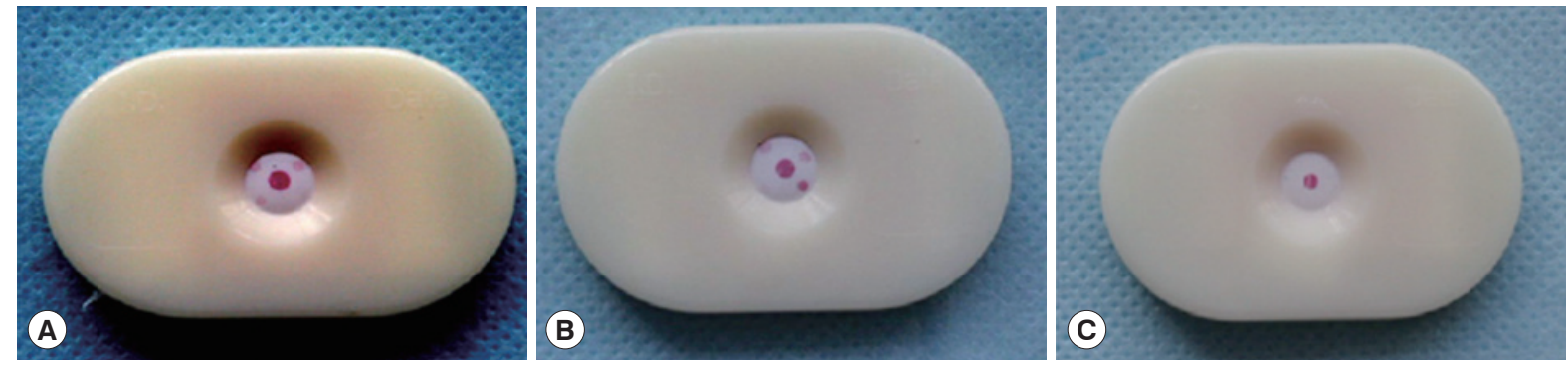

Supplementary Fig. 2. Possible results of the Dot Immunogold Filtration Assay (DIGFA) kit for human echinococcosis. (A) Positive for Echinococcus granulosus. (B) Positive for alveolar echinococcosis. (C) Negative. 